\title{
Relato de experiência acerca do cuidado integral de portadores de sofrimento psíquico na atenção primária de saúde
}

\author{
Experience report on comprehensive care for people with psychological distress in primary \\ health care
}

Informe de experiencia sobre la atención integral a las personas con distrés psicológico en la atención primaria de salud

André Haddad Nascimento ${ }^{1}$, Thais Bassi Cardoso², Henry Luchetti Beatta1, Beatriz Podsclan Rotundo", Gabriela Costa Brito ${ }^{1 *}$.

\section{RESUMO}

Objetivo: Relatar como a atenção primária de saúde pode ser uma facilitadora do reconhecimento, captação e acompanhamento de pacientes com transtornos psiquiátricos, participando de programas de matriciamento e redirecionando o fluxo visando à implementação de uma clínica ampliada e integral. Relato de experiencia: Consiste em uma análise descritiva das atividades realizadas em Unidades Básicas de Saúde de um município do interior de São Paulo, voltadas ao atendimento de pacientes portadores de transtornos psíquicos. As consultas eram conduzidas pelos acadêmicos do curso de medicina, seguidas de discussão dos casos com os membros da equipe da saúde primária, abordando temas como raciocínio clinico, diagnósticos, tratamentos e sobretudo como deveria ser conduzida a reinserção destes pacientes em atividades cotidianos. Considerações finais: A partir dessa reflexão, evidenciou-se a importância da integração de diferentes tipos de serviços para a integralidade do cuidado destinado ao portador de transtornos mentais. Assim como, possibilitou aos acadêmicos a compreensão na prática sobre o funcionamento de programas como o a Política Nacional de Saúde mental, as Redes de Atenção Psicossocial (RAPS), os Centros de Atenção Psicossocial (CAPS) e como estes se correlacionam ao atendimento nas Estratégias de Saúde da Família.

Palavras-chave: Atenção primaria de saúde, Redes de atenção psicossocial, Saúde mental.

\begin{abstract}
Objective: To report how primary health care can be a facilitator of the recognition, recruitment and monitoring of patients with psychiatric disorders, participating in matriculation programs and redirecting the flow aiming at the implementation of an expanded and comprehensive clinic. Experience report: It consists of a descriptive analysis of the activities carried out in Basic Health Units in a city in the interior of São Paulo, focused on the care of patients with mental disorders. The consultations were conducted by medical school students, followed by discussion of cases with members of the primary health team, addressing topics such as clinical reasoning diagnoses, treatments and, above all, how the reintegration of these patients into daily activities should be conducted. Final considerations: From this reflection, it became evident the importance of integrating different types of services for the integrality of care for patients with mental disorders. As well, it made it possible for students to understand in practice the functioning of programs such as the National Mental Health Policy, the Psychosocial Care Networks (RAPS), the Psychosocial Care Centers (CAPS) and how they correlate to care in the Strategies for Family Health.
\end{abstract}

Keywords: Primary health care, Psychosocial care networks, Mental health.

${ }^{1}$ Universidade de Franca (UNIFRAN), Franca - SP. *E-mail: gc_brito@hotmai.com

2 Faculdade de Medicina de Botucatu (FMB), Botucatu - SP.

SUBMETIDO EM: $3 / 2021$ 


\section{RESUMEN}

Objetivo: Informar cómo la atención primaria de salud puede ser un facilitador del reconocimiento, reclutamiento y seguimiento de pacientes con trastornos psiquiátricos, participando en programas de matrícula y reorientando el flujo con miras a la implementación de una clínica ampliada e integral. Informe de experiencia: Consiste en un análisis descriptivo de las actividades realizadas en Unidades Básicas de Salud en una ciudad del interior de São Paulo, enfocadas a la atención de pacientes con trastornos mentales. Las consultas fueron realizadas por estudiantes de la Facultad de Medicina, seguidas de la discusión de casos con miembros del equipo de atención primaria de salud, abordando temas como el razonamiento clínico, diagnósticos, tratamientos y, sobre todo, cómo se debe realizar la reinserción de estos pacientes a las actividades cotidianas. Consideraciones finales: De esta reflexión se hizo evidente la importancia de integrar diferentes tipos de servicios para la integralidad de la atención a los pacientes con trastornos mentales. Asimismo, permitió que los estudiantes entendieran en la práctica el funcionamiento de programas como la Política Nacional de Salud Mental, las Redes de Atención Psicosocial (RAPS), los Centros de Atención Psicosocial (CAPS) y cómo se correlacionan con las Estrategias de Salud de la familia.

Palabras clave: Atención primaria de salud, Redes de atención psicosocial, Salud mental.

\section{INTRODUÇÃO}

Os primórdios do processo de reforma psiquiátrica no Brasil são contemporâneos ao movimento sanitarista nos anos 70, o qual buscava alterações dos modelos de atenção e gestão em saúde, além de estimular a defesa da saúde coletiva, a equidade e igualdade na oferta dos serviços de saúde (HIRDES A, 2009).

O processo de Reforma Psiquiátrica brasileira tem uma história única, inserida em um contexto internacional de mudanças pelo fim do modelo asilar de saúde. Surgindo, ao final dos anos 70 , em meio à crise do modelo de assistência centrado no hospital psiquiátrico, e na emersão dos movimentos sociais pelos direitos dos pacientes psiquiátricos, o processo da Reforma Psiquiátrica brasileira representou um marco para saúde pública e, sobretudo, para os portadores de sofrimento psíquico (AMARANTE P e NUNES MO, 2018).

A reforma psiquiátrica, hoje bem estruturada e consolidada, tem possibilitado transformações nas reflexões sobre a temática da saúde mental, além da implantação de novos modelos de assistência e novas estratégias de cuidado (FARINHA MG e BRAGA TBM, 2018).

Nesse cenário, a desinstitucionalização da loucura tornou-se um dos princípios fundamentos da política de saúde mental, permitindo a substituição dos manicômios por outros serviços que viabilizem a "reinserção" das pessoas com transtornos mentais nos cotidianos da sociedade, possibilitando que os sujeitos, que antes viviam internados em hospitais psiquiátricos, possam contar com um tratamento mais próximo de seus familiares e da comunidade (BARROSO SM e SILVA MA, 2011).

Entretanto, a sociedade, as famílias, e mesmo muitos profissionais de saúde estão pouco preparados e amparados para acolher o portador de sofrimento psíquico (SILVA MP, et al., 2016). Há ainda uma necessidade de esforços para formação e conscientização, tantos dos acadêmicos em formação como dos demais autores da área da saúde para que busquem de forma continuada reverter a lógica de atenção à saúde mental, em que prevaleceu por muitos anos, a exclusão e o preconceito (COSTA-ROSA A, et al., 2000).

Este relato de experiência teve como objetivo exemplificar como a atenção primária de saúde funciona como um facilitador do reconhecimento, captação e acompanhamento de pacientes com transtornos psiquiátricos, participando de programas de matriciamento e redirecionando o fluxo visando à implementação de uma clínica ampliada e integral. Além disso, objetivou a demonstração da importância da inserção de acadêmicos do curso de medicina na rotina do cuidado com a saúde mental, sendo facilitadora do aprendizado e entendimento sobre as rotinas adotadas por meio de programas da Política Nacional de Saúde Mental. 


\section{RELATO DE EXPERIÊNCIA}

Trata-se de uma análise descritiva das atividades realizadas em Unidades Básicas de Saúde durante a disciplina Programa de Integração do Ensino e Saúde da Família (PIESF), prevista na grade curricular do curso de medicina da Universidade de Franca, com foco no atendimento de portadores de transtornos mentais.

Inicialmente o grupo de alunos se reuniram nas dependências da universidade para que fossem ministradas aulas teóricas sobre o processo de reforma sanitária e psiquiátrica pelo qual o país passou nos anos 70. Juntamente, foram introduzidos conceitos importantes para compreensão do funcionamento e deveres das redes de apoio a saúde mental como os Centros de Atenção Psicossocial (CAPS), as Redes de Atenção à Saúde (RAS), e o processo de matriciamento.

Além disso, a partir de aulas expositivas e apresentações de seminários sobre as principais comorbidades psiquiátricas encontradas na atenção primária - suicídio, abuso de substâncias, depressão, ansiedade, psicoses, entre outros - os alunos obtiveram conhecimentos básicos para a identificação e manejo destas patologias.

O ambiente de estágio foi organizado em uma única unidade de atenção primária, com a participação de 6 alunos, divididos em 3 duplas, com carga horaria de quatro horas semanais e duração de 5 semanas, sendo atendidos em média de 3 a 4 pacientes ao dia. Os discentes investiram em conhecer o funcionamento da unidade e associar as práticas adotadas neste local aos preceitos e diretrizes previstas para as APS, além da construção de um vínculo com a equipe de trabalho e reconhecimento dos principais obstáculos apresentados no cuidado da população adscrita.

A partir do conhecimento de uma alta demanda de pacientes da área da saúde mental, foram definidas prioridades de atendimentos e acolhimento. Dependendo das situações abordadas eram necessárias a solicitação de familiares e o acionamento das redes de apoio, CAPS, RAS e ainda da equipe de matriciamento.

As consultas seguiram os padrões de abordagem ao paciente psiquiátrico usando como base as aulas ministradas nas dependências da universidade, sendo que as os atendimentos partiam do conceito da medicina centrada na pessoa e não apenas na doença. Os indivíduos eram questionados sobre os conhecimentos que estes teriam sobre seus problemas de saúde e quais as ideias sugeridas por eles para um planejamento de ação e manejo das intercorrências identificadas.

Após as consultas os casos eram discutidos com a equipe multidisciplinar visando a melhor abordagem ao paciente, de forma que as necessidades desses fossem atendidas, respeitando os Atributos Nucleares, ou ainda designados essenciais, previsto na Atenção Primária a Saúde (APS), sendo estes o acesso aos serviços de saúde, a longitudinalidade por meio do acompanhamento de forma continuada, a integralidade e a coordenação do cuidado.

A experiência foi muito construtiva e rica, permitindo um desenvolvimento, não somente das habilidades técnicas, mas também humanas. Houve desenvolvimento da autonomia e da responsabilidade dos alunos, trazendo reflexões aos alunos no âmbito da humanização do cuidado.

A inserção dos discentes de medicina na rotina das consultas na unidade básica é de extrema importância, pois a partir dessa eles podem se familiarizar com o processo e ainda agregar informações obtidas na realização da atividade, visto que os estudos teóricos muitas vezes não levam em consideração alguns imprevistos que podem ocorrer durante as consultas, sobretudo quando se trata de saúde mental, nos deparamos com pacientes instáveis e de difícil colaboração.

Além disso, por se tratar de uma metodologia ativa, o conhecimento adquirido durante essas atividades é de grande validade quando nos deparamos com a teoria pela primeira vez, facilitando o entendimento e aprendizado. Percebe-se que as vivências oportunizaram a interação ensino-assistência, possibilitando aos acadêmicos relacionar a teoria e a prática em um mesmo eixo, fortalecendo o aprendizado e aprimorando a visão crítico-reflexiva diante da realidade vivenciada. 
O que se busca na formação do profissional da área da saúde, atualmente, é um processo formativo contextualizado, que considere a relação teoria e prática, preparando futuros médicos para enfrentarem os problemas do processo saúde-doença da população.

\section{DISCUSSÃO}

A contribuição da atenção básica na saúde mental é abordada em vários documentos legislativos, normativos e técnicos do SUS, os quais apresentam a APS como serviços primordiais, não hospitalares de atenção à saúde mental (CAMPOS GWS, et al., 2011).O presente trabalho propôs uma reflexão acerca das questões que envolvem o cuidado em saúde mental no âmbito da Atenção Primária a Saúde (APS), de acordo com os pressupostos da Reforma Psiquiátrica e a atual Política de Saúde Mental no Brasil e a importância do conhecimento destes para formação do acadêmico da área da saúde.

A atenção primária à saúde (APS) é caracterizada como o nível do sistema de saúde encarregado pelo acolhimento à população, sendo este eixo norteador na assistência, constituindo a porta de entrada para o sistema de saúde, responsável por oferecer à população os cuidados necessários para suas patologias mais prevalentes, incluindo com isso medidas de prevenção e promoção de saúde (OLIVEIRA MAC e PEREIRA IC, 2013).

Partindo do pressuposto que a continuidade do cuidado é um elemento central de uma atenção primária efetiva, a proximidade entre profissionais de saúde e pacientes, melhoram a qualidade do serviço de saúde oferecido (COSTA CS, et al., 2011). Além disso, onde a saúde mental está integrada como parte desses serviços o acesso melhora, os transtornos mentais são mais facilmente identificados e tratados e as comorbidades e problemas físicos e mentais também são manejadas de forma mais adequada (RANDEMARK NFR, 2009).

Ações de saúde mental no abito da atenção primária devem ser fundamentadas nos princípios do SUS e da reforma psiquiátrica, cujo principal objetivo é a desinstitucionalização, o que pressupõe a manutenção e seguimento do doente mental em seu território, possibilitando a preservação dos vínculos com estes pacientes e suas famílias evitando assim, internações desnecessárias e preferindo esta opção quando se trata de emergências psiquiátricas (CAMPOS GWS, et al., 2011)

Com a consolidação da reforma psiquiátrica houve grande incremento nos atendimentos e acompanhamento das pessoas portadoras de sofrimento psíquico, fazendo com que a atenção primária aprimorasse a escuta qualificada, efetivando nesse sentido princípios do SUS fundamentais no desenvolvimento de práticas como integralidade e resolubilidade das adversidades encontradas.

As atividades planejadas durante o estágio da disciplina Programa de Integração do Ensino e Saúde da Família (PIESF) previam novos pacientes e também indivíduos já em acompanhamento, objetivando alcançar o primeiro Atributo Nuclear previsto nas diretrizes das APS, a partir do acesso ou primeiro contato, aos adscritos na área da atuação da unidade e, respeitando também a utilização do serviço como fonte de cuidado a cada novo problema ou novo episódio de uma mesma patologia.

O acompanhamento dos portadores de transtornos psíquicos visa também, a longitudinalidade a partir do atendimento continuado ao longo do tempo permite a observação da evolução desses pacientes, assim como a adequação terapêutica necessária e o auxílio para reinserção ou progressão dessas pessoas no cotidiano familiar e social. Oferecendo não apenas o cuidado a saúde mental, mas também, por meio da integralidade, a abertura de todo um leque de serviços oferecidos pela atenção primaria de saúde, como o cuidado biopsicossocial, ações de promoção, prevenção, além de cura para determinadas moléstias e reabilitação (CECÍLIO LCO, 2001).

Contando ainda, com o apoio matricial a partir dos Núcleos de Apoio à Saúde da Familia (NASF), com os CAPS e RAS, como suporte técnico aos serviços de atenção básica e a principal estratégia de qualificação da APS para atender às demandas de saúde mental da população (DELFINI PSS, 2009). Uma equipe técnica responsável pelo apoio matricial compartilha casos com equipes de saúde da família, por meio de discussões conjuntas de casos, intervenções junto às famílias e comunidades ou atendimentos compartilhados (DIMENSTEIN MDB, et al., 2009). 
O matriciamento representa uma ferramenta de auxílio para a coordenação do cuidado do doente psiquiátrico, constituindo um arranjo organizacional que visa fornecer suporte técnico em áreas especificas às equipes responsáveis pelo desenvolvimento de ações de saúde e evitando encaminhamentos excessivos, pois objetiva a capacidade resolutiva de problemas de saúde pela equipe primária, estimulando a interdisciplinaridade e a ampliação da clínica em equipe, o que resgata e valoriza outras dimensões do cuidado além da biológica e dos sintomas, tornando o atendimento singular a cada caso (DIMENSTEIN MDB, et al., 2009).

Outro ponto abordado e praticado durante as consultas corresponde ao quarto e último atributo nuclear da atenção primária, a coordenação do cuidado, por meio da qual as APS têm a função de encaminhar pacientes, caso necessário, para serviços de maiores complexidades, com a garantia de retorno destes para continuidade do cuidado (MENDES EV, 2011).

Por todas as mudanças ocorridas a implementação da reforma psiquiátrica nos anos 70 e junto a ela a reorganização do serviço público de saúde primária, conduz a uma reorientação na formação dos profissionais de saúde para que sejam capazes de atuar sob as novas perspectivas da promoção de saúde, do trabalho em equipe e da medicina humanizada. Tais mudanças apontam para uma necessidade do trabalho em equipe como recurso importante para possibilitar ações que possam atingir a dimensão da integralidade da assistência como um dos eixos estruturais da saúde pública, o que só podem acontecer por meio da integração do ensino nos serviços da comunidade, sobretudo no atendimento à saúde primária.

Partindo do conceito de formação generalista, os discentes e integrantes da Atenção Básica precisam de espaços que facilitem o desenvolvimento de competências, habilidades e atitudes, afim de oferecerem cuidados básicos, identificar comorbidades no âmbito da saúde mental e reconhecer a complexibilidade de cada caso. O entendimento da consulta funciona como ferramenta central para a resolução da demanda em saúde psíquica e o trabalho em conjunto das redes de apoio a portadores dessas comorbidades tornam as intervenções mais eficiente e resolutiva, os pacientes se sentem mais seguros e amparados, além de permitir a construção coletiva de saberes e cuidados.

\section{REFERÊNCIAS}

1. AMARANTE P, NUNES MO. A reforma psiquiátrica no SUS e a luta por uma sociedade sem manicômios. Ciência \& Saúde Coletiva, 2018; 23(6): 2067-2074.

2. BARROSO SM, SILVA MA. Reforma Psiquiátrica Brasileira: o caminho da desinstitucionalização pelo olhar da historiografia. Rev. SPAGESP, 2011; 12(1): 66-78.

3. BRASIL. Ministério da Saúde (MS). Saúde mental no SUS: acesso ao tratamento e mudança do modelo de atenção. Relatório de Gestão 2003-2006. Brasília: Ministério da Saúde (MS), 2007.

4. BRASIL. Ministério da Saúde (MS). Portaria n. 154, de 24/01/2008, Cria os núcleos de apoio à saúde da família. Brasília: Ministério da Saúde (MS); 2008.

5. CAMPOS GWS. Saúde pública e saúde coletiva: campo e núcleo de saberes e práticas. Revista Ciência \& Saúde Coletiva, 2000; 5(2): 219-230.

6. CECÍLIO LCO. As Necessidades de saúde como conceito estruturante na luta pela integralidade e equidade na atenção em saúde. Ciência \& Saúde Coletiva, 2001; 113-126.

7. COSTA CS, et al. A percepção de pacientes e familiares sobre os resultados do tratamento em serviços de saúde mental. Cadernos de Saúde Pública, 2011; 27(5): 995-1007.

8. COSTA-ROSA A. O modo psicossocial: um paradigma das práticas substitutivas ao modo asilar. Loucura \& Civilização collection, 2000; 141-168.

9. DELFINI PSS, et al. Parceria entre CAPS e PSF: o desafio da construção de um novo saber. Ciência \& Saúde Coletiva, 2009; 14(1): 1483-1492.

10. DIMENSTEIN MDB, et al. O apoio matricial em Unidades de Saúde da Família: experimentando inovações em saúde mental. Saúde e Sociedade, 2009; 18(1): 63-74.

11. FARINHA MG, BRAGA TBM. Sistema único de saúde e a reforma psiquiátrica: desafios e perspectivas. Revista da Abordagem Gestáltica, 2018; 24(3): 366-378.

12. FONSECA MLG, et al. Sofrimento difuso e transtornos mentais comuns: uma revisão bibliográfica. Revista Atenção Primária a Saúde, 2008; 11(3):285-294.

13. HIRDES A. A reforma psiquiátrica no Brasil: uma revisão. Ciência \& Saúde Coletiva, 2009; 14(1): $297-305$.

14. Macedo JP, et al. A regionalização da saúde mental e os novos desafios da Reforma Psiquiátrica brasileira. Saúde Soc., 2017; 26(1): 155-70.

15. MENDES EV. As redes de atenção à saúde. 2ª ed. Brasília: OPAS, 2011 
16. OLIVEIRA MAC, PEREIRA IC. Atributos essenciais da Atenção Primária e a Estratégia Saúde da Família. Revista Brasileira de Enfermagem, 2013; 66(1): 158-164.

17. MINOZZO F, COSTA II. Apoio matricial em saúde mental entre CAPS e Saúde da Família: trilhando caminhos possíveis. Psico-USF, 2013; 18(1): 151-60.

18. RANDEMARK NFR. Reabilitação psicossocial de pessoas com transtorno mental no contexto da reforma psiquiátrica brasileira: representações das famílias. 2006. 270 f. Tese (Doutorado em Enfermagem Psiquiátrica) - Escola de Enfermagem, Universidade de São Paulo, São Paulo. 2009.

19. SAMANTA ML, CARMAGNANI MI. Programa de saúde da família no Brasil: um enfoque sobre seus pressupostos básicos, operacionalização e vantagens. Saúde e Sociedade, 2001; 10(1): 33-54.

20. SHIMIZU HE, CARVALHO JD. O processo de trabalho na Estratégia Saúde da Família e suas repercussões no processo saúde-doença. Ciênc. Saúde Colet., 2012; 17(9): 2405-14.

21. SILVA MP, et al. Relação entre saúde mental e trabalho: a concepção de sindicalistas e possíveis formas de enfrentamento. Revista Brasileira de Saúde Ocupacional, 2016; 41(1): 23-41. 